\title{
Robótica Educativa. La programación como parte de un proceso educativo.
}

\author{
Educational Robotics. Programming as part of a educational process. \\ José Miguel García \\ Facultad Latinoamericana de Ciencias Sociales. FLACSO. Uruguay. \\ jgarcia@flacso.edu.uy
}

\begin{abstract}
Resumen
El presente artículo aborda la temática de la Robótica Educativa en Uruguay. Presenta una breve reseña del estado del arte, así como de distintos enfoques con que es abordada. Se analiza la robótica educativa como una forma de trabajo que sustenta el desarrollo del pensamiento computacional en niños, niñas y jóvenes de educación general, más allá de la programación, y de las orientaciones profesionales a las que se dediquen en el futuro.

Se presenta la programación como una fase del trabajo en proyectos específicos elegidos por los estudiantes, en una dinámica de cuatro palabras: Imaginar, Diseñar, Construir y Programar, que componen el continuo que representa la robótica educativa y su forma de trabajo en la enseñanza primaria y media.
\end{abstract}

\section{Palabras Clave}

Robótica educativa, Plan Ceibal, Programación

\begin{abstract}
This article addresses the topic of Educational Robotics in Uruguay. It includes a brief review of the history of the discipline in this country, as well as the different approaches that it presents. The Educational Robotic is analyzed as a way of working that supports the development of computational thinking in children and young people in general education, beyond the programming and the profession that they will be choose in the future.

It presents the programming as a stage in the development of specific projects, chosen by the students. Imagine, design, build and programming make up the continuum that represents educational robotics and its way of working in elementary and high school.
\end{abstract}

\section{Keywords}

Educational Robotics, Ceibal Plan, Programming

\section{Introducción}

Los procesos de incorporación de tecnologías en la educación en el Uruguay datan de hace más de 30 años y han recorrido un largo trayecto, desde las primeras experiencias desarrolladas en la década de los '80, fundamentalmente con el uso de los "home computer" hasta la situación actual, donde el modelo de un computador por alumno está generalizado en el ámbito de toda la educación pública, tanto primaria como media. 
Las primeras experiencias de trabajo con elementos externos a las computadoras, como lo es la robótica, comenzaron a desarrollarse en nuestro país a principios de los '90, mediante el uso de dispositivos desarrollados localmente y especialmente diseñados para fines educativos, debido a la escasez y alto costo de los sistemas importados. Así, se desarrollaron prácticas de robótica educativa con materiales de diverso origen en talleres para alumnos de educación primaria y media, que fueron controlados con los primeros modelos de computadoras personales como lo fueron las XT. En la figura 1 puede verse el equipo de trabajo y el proyecto de un cebador de mate automatizado, realizado por alumnos de $4^{\circ}$ año de enseñanza secundaria (15-16 años) en 1994.

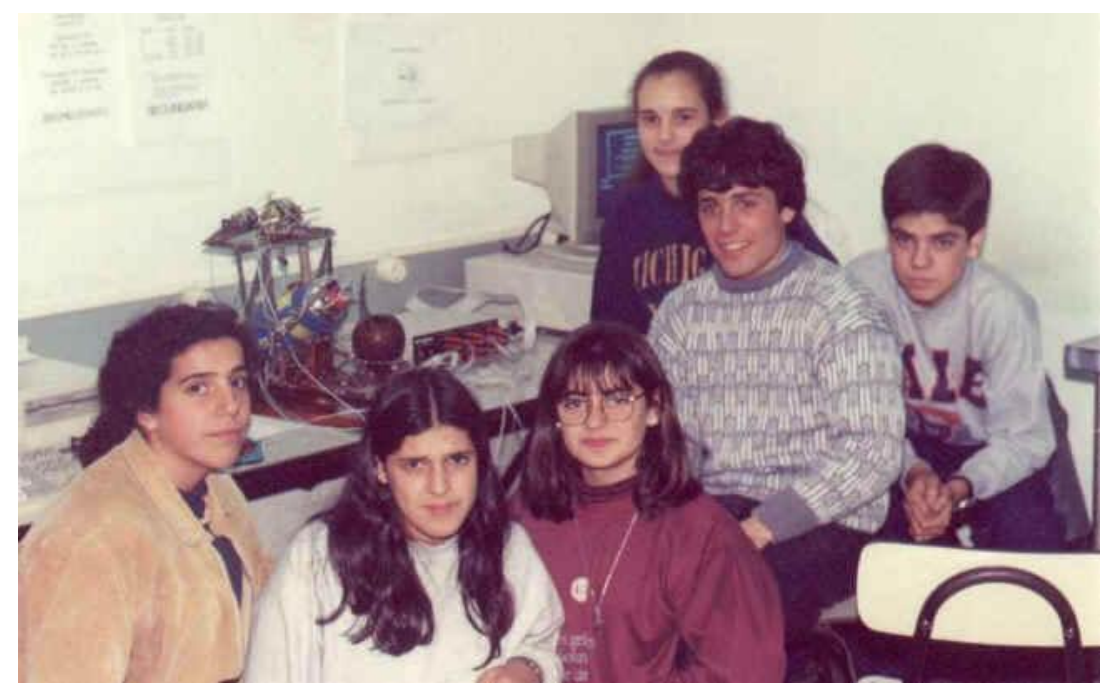

Figura 1: Grupo de desarrollo de "Ansina: cebador de mate”, $1994^{1}$

Los robots utilizaban una interface de desarrollo nacional y el control se realizaba desde la computadora a través de lenguajes de programación como Turbo Pascal, Logo Writer o WinLogo. Este sistema permitía controlar 4 salidas de voltaje fijo, 4 de voltaje regulable e inversión de marcha y contaba con capacidad para trabajar con 4 sensores.

En esa época las ventajas de realizar este tipo de experiencias no fueron visibilizadas y éstas, que se desarrollaron casi exclusivamente en el ámbito de la educación privada, no se expandieron con rapidez. Fueron pocos los centros educativos que desarrollaron estas prácticas durante los 15 años previos a la implementación del modelo 1 a 1 en Uruguay, que fueron enfocados en la línea que presentan López y Andrade (2013):

"Es posible entonces utilizar la robótica como elemento motivador e integrador del conocimiento de las diferentes áreas y para esto son útiles diversas plataformas robóticas que se consiguen en el comercio o mediante la construcción del robot con elementos nuevos o de reciclaje." (López y Andrade: 2013)

La implementación del modelo de un computador por alumno en Uruguay, denominado Plan $\mathrm{Ceibal}^{2}$ fue iniciado en el año 2007, completando la entrega de laptops a los alumnos y docentes de todas las escuelas públicas de enseñanza primaria en 2009. La expansión a educación media comenzó en el año 2010, completando la universalización en el año 2014.

\footnotetext{
${ }^{1}$ Más información de este proyecto en http://www.argos.edu.uy/sitio/proyectos/robotica_cebador_de_mate.html\#proyecto

${ }^{2}$ Plan Ceibal: Nombre local del proyecto de un computador por alumno en Uruguay. http://ceibal.edu.uy/
} 
Esta distribución generalizada, sumada con las capacidades técnicas y la baja de precios en desarrollos tecnológicos provocó un gran salto en las prácticas de la robótica educativa en nuestro país, pasando al ámbito de la educación pública. Estas iniciaron con el proyecto denominado "Robótica Aplicada a la Enseñanza Secundaria", que distribuyó equipamiento en diversos centros educativos, y el Proyecto Butiá ${ }^{3}$, desarrollado desde el Instituto de Computación de la Facultad de Ingeniería, Universidad de la República, que a partir del año 2009 genera equipamiento de bajo costo y en formato de hardware libre, teniendo un fuerte desarrollo en diversos centros educativos de enseñanza secundaria y primaria. Desde el propio Plan Ceibal se implementa en 2010 un piloto, y a partir del año 2011 se masifica la distribución de kit a los centros educativos de enseñanza media, así como de escuelas primarias de tiempo completo y tiempo extendido.

El momento actual es, por lo tanto, propicio para el desarrollo de la robótica educativa.

\section{Enfoque de las cuatro palabras}

Es necesario establecer algunas ideas clave acerca de lo que entendemos por robótica educativa. En primer lugar es importante diferenciarla de la robótica industrial, en el entendido que en ésta la clave de éxito está en el producto final, de manera que el dispositivo creado funcione de acuerdo a los requerimientos y expectativas de quien lo adquiere. En la robótica educativa la clave está en el proceso de desarrollo del robot, más allá de que su aplicabilidad o funcionamiento no resulten de gran trascendencia.

Reconocemos en el proceso de trabajo cuatro líneas o palabras, que entendemos enmarcan estas prácticas: Imaginar, Diseñar, Construir y Programar (García y Castrillejo: 2007).

Imaginar: los alumnos imaginan y debaten sobre ideas de dispositivos que podría interesarles construir. Para esto, se pone énfasis en la importancia de imaginar dispositivos que resuelvan problemas concretos. De esta manera se pone en juego la creatividad de los alumnos, tanto en la realización de un análisis de las posibilidades de trabajo como en la propuesta de soluciones específicas a problemáticas que pueden detectar o imaginar.

Diseñar: Una vez acordada la temática a trabajar, es necesario realizar un diseño más concreto del dispositivo a crear, lo que implica una fuerte vinculación entre la imaginación y el mundo físico, donde procuran plasmar no sólo cuáles serán las potencialidades del artefacto, sino cómo se construiría. Esta etapa favorece el proceso de plasmar ideas en proyectos, a través de un ida y vuelta entre imaginación y capacidad de concreción. Esto provoca también la necesidad de recurrir a conocimientos previos o de investigar en función de lo que se necesita para el desarrollo. La figura 2 presenta el diseño de un dispositivo para sellado de hojas realizado por docentes y estudiantes de profesorado en el Centro Regional de Profesores del Norte.

\footnotetext{
${ }^{3}$ Sitio oficial del proyecto en http://www.fing.edu.uy/inco/proyectos/butia/
} 


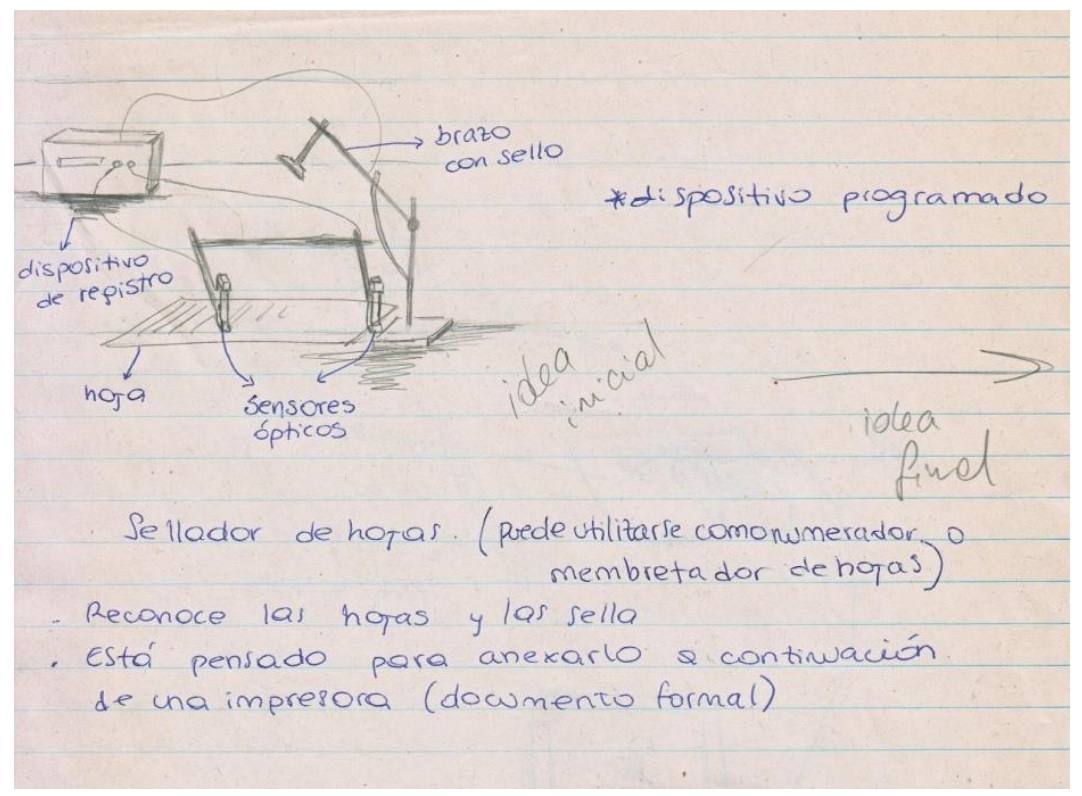

Figura 2: Diseño de robot "Sellador" en jornadas del CERP Rivera

Construir: Los proyectos imaginados y diseñados son luego armados por el equipo de trabajo, conjugando los saberes teóricos con las habilidades manuales necesarias para su desarrollo. Se reivindica aquí el trabajo con las manos como parte del proceso creativo.

Programar: Los mecanismos construidos son programados a través de la computadora. Esto desarrolla tanto pensamiento lógico como capacidad de autopercepción y análisis espacial. Para poder indicarle al robot los movimientos que se quieren realizar es necesario conceptualizarlos, muchas veces con el propio cuerpo, así como anticipar qué ocurrirá con el mecanismo cuando se ejecuten las órdenes dadas a través del programa. La programación en esta modalidad es motivada por el interés de que el artefacto realice las tareas para las que fue diseñado.

Si bien en un principio estas acciones se van desarrollando como un proceso, este nunca es lineal. Estas cuatro palabras no definen cuatro pasos diferenciados, sino que interactúan constantemente entre sí, pues los diseños, las construcciones y la programación van modificando las ideas y propuestas originales.

A modo de ejemplo, la figura 3 representa el proyecto mostrado en la figura 2 , en su etapa final: 


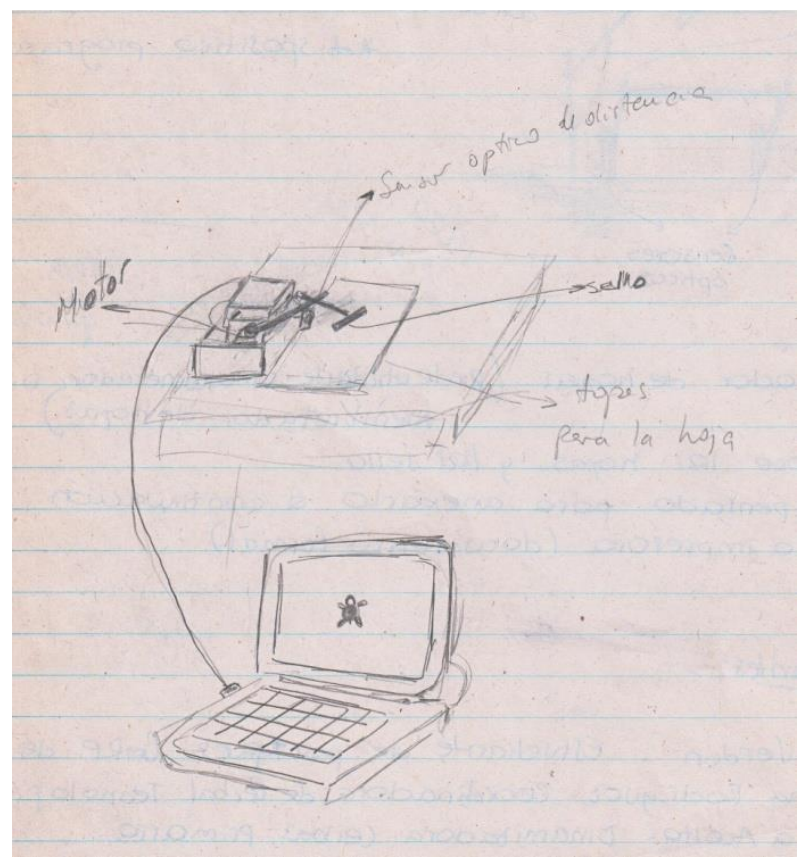

Figura 3: Dibujo del proyecto como fue construido en CERP Rivera

La figura 4 muestra la fotografía del artefacto final de este proyecto:



Figura 4: Dispositivo sellador final. CERP Rivera

\section{Distintos modelos.}

Si bien hemos referido a un enfoque de la robótica educativa a partir de proyectos creados por los participantes, la gran variedad de dispositivos disponibles permite trabajos basados en distintos formatos. El más contrapuesto al presentado en el apartado anterior sería el armado de 
un robot siguiendo específicamente las indicaciones de los manuales correspondientes, incluyendo las de la propia programación.

Para clasificar los distintos enfoques de trabajo en robótica educativa podemos usar el esquema propuesto en García y Castrillejo (2011. Pp. 315), donde se presentan las cuatro palabras como vértices de un tetraedro. En la figura 5 se reproduce este esquema.

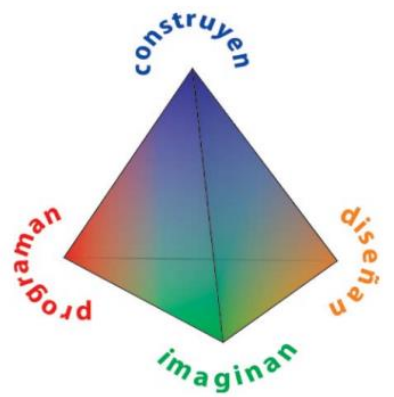

Figura 5: Cuatro palabras para Robótica Educativa. Fuente: García y Castrillejo 2011

El trabajo con robots prefabricados, siguiendo manuales de armado, inclina la experiencia hacia alguno de los vértices quitando la riqueza de la imaginación o el diseño, en los casos más favorables. Puede ocurrir también que todo el trabajo esté reflejado en los manuales y que sólo se sigan las indicaciones que éstos presentan, en cuyo caso la creatividad queda vedada.

Entendemos que si bien puede resultar positiva la utilización de este tipo de modelos para las primeras instancias de autoaprendizaje, resultará en el uso de juguetes sofisticados si se persiste en este tipo de esquemas de trabajo.

Hay diversos ejemplos tecnologías disponibles enfocadas fuertemente a la programación en el ámbito de la robótica educativa. Sin desmerecer esta línea, el trabajo con este tipo de dispositivos puede fortalecer aspectos valiosos en los espacios de aprendizaje, pero poniendo el centro en el control de la tecnología propuesta más que en los desarrollos de proyectos particulares por parte de los usuarios.

Compartimos el planteo de Urrea (2001) acerca de la importancia de trabajar en proyecto creados por los propios alumnos:

"Ellos deberían ser capaces de escoger las ideas que ellos quisieran explorar, los recursos que quisieran incluir en sus experimentos, solucionar los retos, discutir y presentar sus proyectos finales entre ellos mismos y con otros miembros de la comunidad. Aprendizaje de inmersión se refiere a la noción de que los alumnos estén inmersos en un proceso de aprendizaje, y dispongan de mucho tiempo para dedicarse al juego y a explorar a fondo sus ideas." (Urrea, 2001)

En esta línea, la utilización de materiales no incluidos en los kits puede proporcionar buenas alternativas, dando mayor amplitud a la hora de imaginar y diseñar artefactos, sin quedarse limitado a los componentes entregados por los fabricantes. Si bien la utilización de motores y sensores está condicionada por el dispositivo de control o interface, el resto de la construcción puede no quedar tan atada al material proporcionado en lis kits.

A modo de ejemplo, destacamos una compactadora de basura en una papelera, desarrollada en un taller por estudiantes de formación docente que se iniciaban en la temática, que se muestra en la figura 6. 


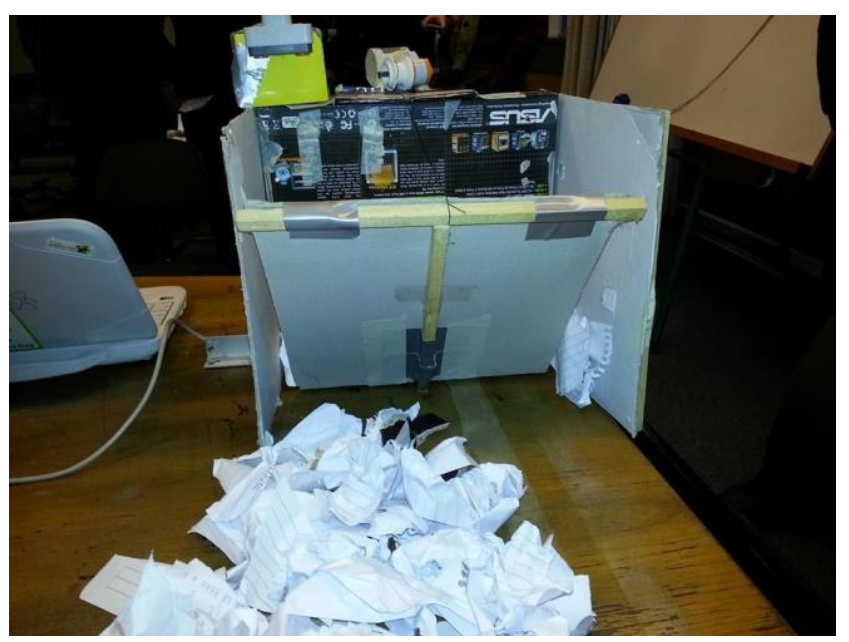

Figura 6: Papelera con compactadora

De ser necesario pueden también utilizarse otro tipo de dispositivos para la adquisición de datos que, en programas abiertos como el TortuBot, permiten interactuar con los kits disponibles. En el caso de Uruguay, y con la amplia distribución de equipos XO, el uso de la entrada de micrófono del laptop amplifica la disponibilidad de sensores, tal como se describe en la página que al respecto se publica en el sitio web de Sugar Labs. ${ }^{4}$

\section{Más allá de la robótica}

Entendemos la robótica educativa como un proceso de aprendizaje que trasciende las particularidades de aquellos estudiantes que se "perfilen" hacia la programación o las tecnologías. Compartimos los planteos de Zapata-Ros respecto a la importancia del desarrollo del pensamiento computacional en el marco de una educación generalizada.

"Por tanto, como idea-fuerza, es fundamental que, al igual que sucede con la música, con la danza o con la práctica de deportes, se fomente una práctica formativa del pensamiento computacional desde las primeras etapas de desarrollo. Y para ello, al igual que se pone en contacto a los niños con un entorno musical o de práctica de danza o deportiva,... se haga con un entorno de objetos que promuevan, que fomenten, a través de la observación y de la manipulación, aprendizajes adecuados para favorecer este pensamiento" (Zapata-Ros, 2014).

Tomando su ejemplo, y de la misma manera que se trabaja con música con todas y todos, y no solamente con aquellas personas que se dedicarán a ella en forma profesional, entendemos que el trabajo con robótica educativa debe ser considerado para todas y todos, en el marco de una educación general. Por alguna razón estas prácticas aún son vistas como propicias solamente para alumnos con inclinaciones hacia la tecnología.

Entendemos que la robótica educativa entonces no debe ser vista como privativa para los alumnos que trabajarán en áreas técnicas, sino como dispositivo pedagógico para un aprendizaje creativo.

Dentro de los múltiples aprendizajes que provocan estas prácticas destacamos también la importancia de que los alumnos se conviertan en desarrolladores de tecnología, lo que les permitirá desarrollar una visión crítica de la misma. En los países en desarrollo, donde las

\footnotetext{
${ }^{4}$ https://wiki.sugarlabs.org/go/Activities/Turtle_Art/Uso_de_Tortuga_Arte_Sensores\#Enlaces
} 
tecnologías más visibles ${ }^{5}$ son importadas, el hecho de que los alumnos descubran que pueden hacer desarrollos tecnológicos innovadores con sus propias manos y mentes aporta un gran diferencial.

Otro componente valioso de este tipo de trabajo en el aula es el aprendizaje a través del desarrollo de proyectos. Si las etapas de imaginación y diseño se realizan con los alumnos y se trabaja en proyectos elegidos por ellos se generan procesos altamente creativos, favoreciendo el aprendizaje colaborativo en un proceso grupal. Son, por tanto, más motivadores que aquellos que son impuestos o que son desarrollados para "complacer" al docente que los plantea.

A modo de ejemplo, la figura 7 muestra el desarrollo de un dispensador de medicación para personas con Alzheimer, creado por un grupo de estudiantes de tercer año de enseñanza media (14-15 años). El mismo estaba programado para que a las horas específicas en que la persona debía tomar su medicación, el dispositivo la entregara. La idea original surgió de la situación particular de una alumna que tenía a su abuela con esa enfermedad. La pérdida de la memoria, característica de esta dolencia, le impedía tomar la medicación con regularidad. Así, el grupo se dedicó a diseñar una solución concreta a esta problemática particular, que les resultaba propia y motivadora, independientemente de que siempre tuvieron claro que por sus capacidades técnicas y los tiempos con que contaban, este aparato no sería usado en forma sistemática.

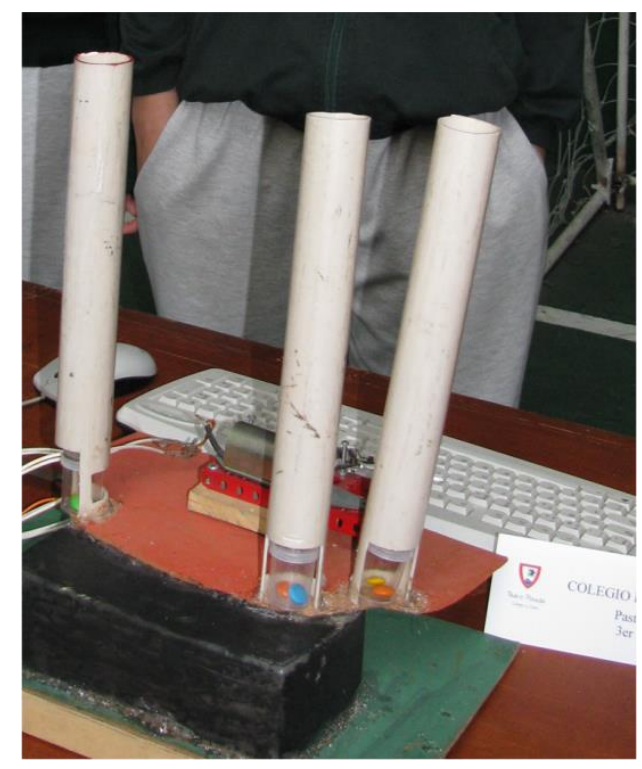

Figura 7: Dispensador robotizado de medicación para personas con Alzhéimer

El desarrollo de proyectos de robótica permite asimismo el aprendizaje a partir del error, ya que los diseños originales nunca concuerdan con las versiones finales. En este caso, el error más que un elemento a evitar se convierte en un factor importante como motor de aprendizajes. Así, el error no es castigado, sino tomado como base y disparador de procesos de aprendizaje.

\section{Codificar es más que programar.}

El trabajo en robótica educativa puede ir más allá del desarrollo tecnológico. Cuando se realiza una animación, o un cuento, a través de dispositivos como la robótica y la programación, se está codificando mucho más que un programa informático. Se está codificando también el propio

\footnotetext{
${ }^{5}$ Algunos desarrollos tecnológicos nacionales como el diseño y fabricación de marcapasos que son exportados a países desarrollados son poco conocidos en este país.
} 
lenguaje a través de dispositivos tecnológicos. Un excelente ejemplo de esta visión la aportó en Montevideo la profesora Aura Mora (Colombia), quien realizó un taller con alumnos de una escuela primaria en su visita a nuestro país. La consigna consistió en plantear a los alumnos que desarrollaran una historia que luego representaron a través de un dispositivo robótico que diseñaron. En la figura 8 puede observarse el guión de la historia que realizaron, acerca de la visita de un extraterrestre, así como la representación robótica que generaron y el programa codificado en TortuBot ${ }^{6}$, una aplicación basada en TortugArte ${ }^{7}$ y desarrollada en Uruguay por el Instituto de Computación de la Facultad de Ingeniería de la Universidad de la República.

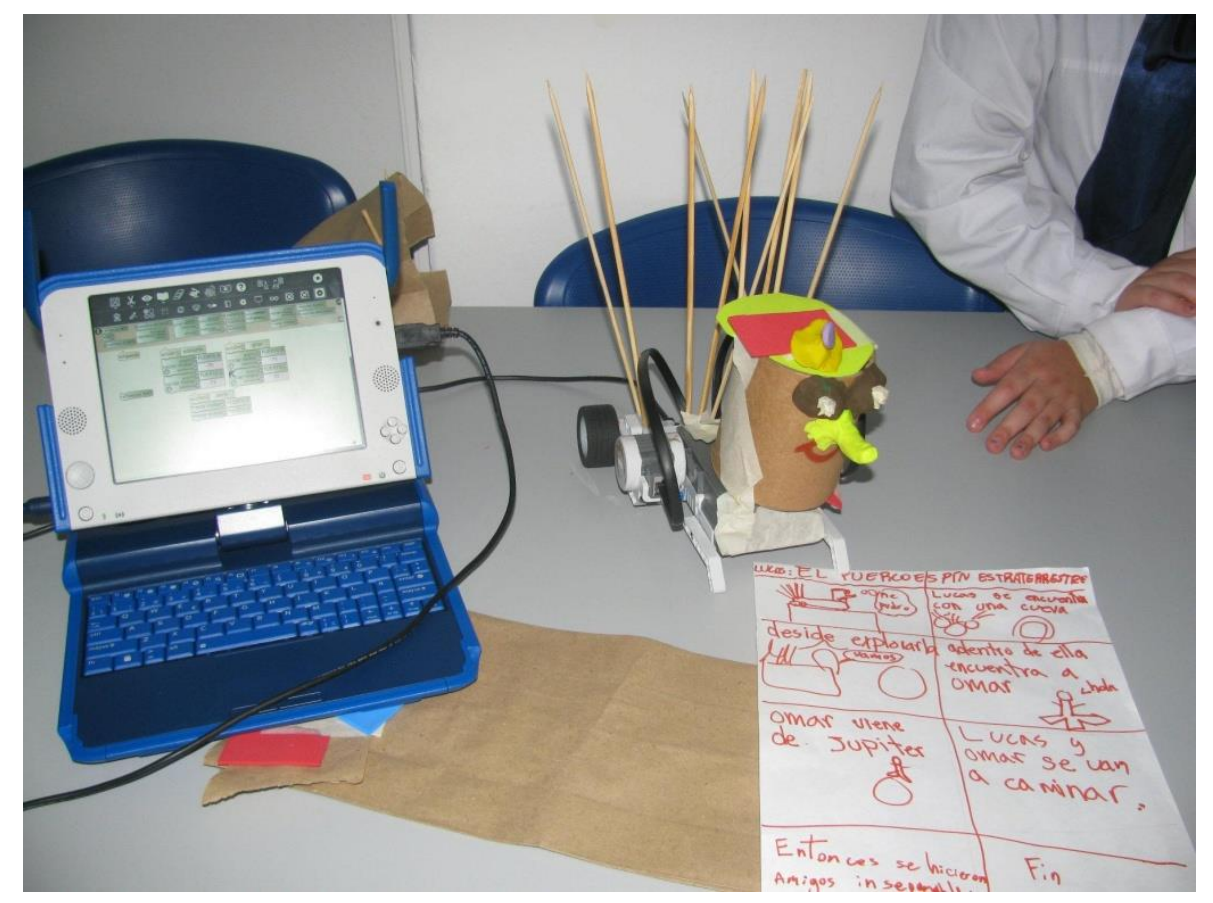

Figura 8: Proyecto desarrollado en el marco del taller con la profesora Aura Mora, Montevideo 2013

Otra experiencia significativa en esta línea fue la realizada en conjunto entre un profesor del taller de robótica y una profesora de inglés, hace ya unos cuantos años. La propuesta, dirigida a aquellos alumnos a los que les resultara interesante, consistía en la selección de un libro en inglés, su lectura, y la posterior construcción de un dispositivo robotizado que representara alguna de las ideas del texto. Aquí, la robótica fue motivadora de lectura e interpretación de textos en una lengua extranjera, además del trabajo con el propio mecanismo. Aquí se puede ver claramente que la codificación no es exclusiva de la programación, sino del lenguaje en distintos idiomas: el inglés, el español, el de programación, el de construcción de máquinas, el de interpretación de los datos que aportan los sensores, etc.

\section{Docentes aprendices}

En formatos abiertos de trabajo con robótica educativa, donde los alumnos imaginan, diseñan, construyen y programan sus propios robots, el docente asume un rol de aprendiz, sumándose al

\footnotetext{
${ }^{6}$ https://www.fing.edu.uy/inco/proyectos/butia/mediawiki/index.php/TortuBots

${ }^{7}$ http://wiki.sugarlabs.org/go/Activities/Turtle_Art/lang-es
} 
grupo de trabajo. Si bien es necesario que sea un componente activo en los desarrollos, a diferencia de los modelos educativos tradicionales, el docente no siempre podrá anticiparse a todas las formas de solucionar problemas durante los procesos de innovación. Aunque el docente pueda tener más experiencia que sus alumnos, no será capaz de predecir las dificultades que se encontrarán al momento de desarrollar un proyecto que no ha sido trabajado con anterioridad. De la misma forma no habrá una manera única de resolver los problemas planteados, por lo que la apertura hacia el aprendizaje del docente, permitiendo experimentar a los alumnos diversas alternativas resultará clave para un trabajo conjunto. Así se está participando de un espacio donde el docente aprende a aprender, lo que se transforma en una herramienta valiosa ya que los alumnos pueden visualizarlo como un par. Entendemos que los alumnos aprenderán más de las estrategias de aprendizaje que desarrolla el docente que de los conocimientos específicos que pueda "trasmitirles". Concordamos con Papert que

"La mejor forma de llegar a ser un buen carpintero es participando con un buen carpintero en el trabajo de la carpintería. Por analogía, la manera de llegar a ser un buen aprendiz es participar con un buen aprendiz en el acto de aprendizaje. En otras palabras, el estudiante debería encontrar un profesor-aprendiz con quien compartir el acto de aprendizaje. Pero esto rara vez ocurre en la escuela, debido a que el docente ya sabe lo que se está enseñando y, en consecuencia, no puede estar aprendiendo de manera auténtica. Lo que yo veo como una parte esencial de la experiencia de Logo es esta relación de ser aprendiz en el aprendizaje.” (Papert: 2001)

Así los alumnos aprenderán a aprender, a través de un espacio de aprendizaje compartido con el docente.

\section{A modo de cierre.}

Entendemos que codificar no es sólo programar, y que no es algo exclusivo de los programadores ni de quienes se dedicarán a ello en el futuro. Trabajar con programación y robótica no puede ser exclusivamente un dispositivo creado o desarrollado para incentivar a los estudiantes a dedicarse a carreras del área de la ingeniería. El trabajo en torno a las cuatro palabras que describimos (imaginar, diseñar, construir y programar) conforma un conglomerado de acciones y actividades que potencian aprendizajes no compartimentados. Estos pueden ser enfocados con distintas modalidades, orientadas a la construcción, la programación o la comunicación en sus diferentes acepciones, en espacios abiertos que vayan más allá de los marcos tradicionales de enseñanza, poniendo más énfasis en los alumnos y en sus aprendizajes que en los contenidos a trasmitir desde una "autoridad" centralizada en el docente.

Presentación del manuscrito: 29 de julio de 2015

Fecha de aprobación: 23 de agosto de 2015

Fecha de publicación: 15 de septiembre de 2015

García, J. M. (2015). Robótica Educativa. La programación como proceso. RED. Revista de Educación a Distancia, 46(8). 15 de Septiembre de 2015. Consultado el (dd/mm/aa) en http://www.um.es/ead/red/46 


\section{Referencias}

García, J. M.; y Castrillejo, D. (2007). Robótica en la escuela del Tercer Mundo. Una manera diferente de aprender a aprender. Montevideo: Consultado el 10/07/2015 en http://www.argos.edu.uy/sitio/documentos/Robotica_en_la_escuela.pdf

García, J. M.; y Castrillejo, D. (2011). Los Robots como excusa, en El modelo CEIBAL. Nuevas tendencias para el aprendizaje". Montevideo: ANEP-CEIBAL, pp. 300-333. Consultado el 10/07/2015 en http://www.anep.edu.uy/anep/phocadownload/Publicaciones/Plan_Ceibal/el\%20modelo\%20 ceibal\%20nuevas\%20tendencias\%20para\%20el\%20aprendizaje.pdf

López, Pedro y Andrade, H (2013) Aprendizaje con robótica, algunas experiencias. Educación, vol. 37, núm. 1, enero-junio, 2013, pp. 43-63, Universidad de Costa Rica, San Pedro, Montes de Oca, Costa Rica. Consultado el 10/07/2015 en http://www.redalyc.org/pdf/440/44028564003.pdf

Papert, S. (2001): ¿Qué es Logo? ¿Quién lo necesita? En módulo Programación en la Educación Escolar, Eduteka. Consultado el en http://www.eduteka.org/modulos.php?catx $=9 \&$ idSubX $=288$

Urrea, C. (2001): Diseño de aparatos robóticos: colaboración para el aprendizaje entre escuela rural y comunidad. En módulo Programación en la Educación Escolar, Eduteka. Consultado el 10/07/2015 en http://www.eduteka.org/modulos/9/292/15/1

Zapata-Ros, M. (2014). ¿Por qué “pensamiento computacional”? (I), Consultado el 10/07/2015 en http://redesabiertas.blogspot.com.es/2014/11/por-que-pensamiento-computacional-i.html $[12 / 02 / 2015]$

Esta obra está bajo una Licencia Creative Commons Atribución-NoComercial-SinDerivar 4.0 Internacional.

El autor del presente artículo se hace responsable por lo que expresa, lo cual no necesariamente refleja la opinión de la Revista RED ni de las organizaciones en las que se desempeña. 